\title{
Semi-stochastic cell-level computational modelling of cellular forces: application to contractures in burns and cyclic loading
}

\author{
F. J. Vermolen - A. Gefen
}

Received: 25 August 2014 / Accepted: 28 February 2015 / Published online: 17 March 2015

(C) The Author(s) 2015. This article is published with open access at Springerlink.com

\begin{abstract}
A phenomenological model is formulated to model cellular forces on extracellular material. The model is capable of modelling both expansion and contractile forces. This work is based on the assumption of linear elasticity, which allows a superposition argument to arrive at fundamental expressions for cellular forces. It is also shown how the cellular forces can be implemented using different strategies, as well as an extension to cellular point sources. Illustrations are given for modelling a (permanent) contraction (e.g. a contracture) of burns and for cyclic loading by the cells.
\end{abstract}

Keywords Cellular forces - Cell-level modeling - Semistochastic model $\cdot$ Burns $\cdot$ Contractors

\section{Introduction}

Many biological processes where cells play an important role, such as wound healing, tumour growth, angiogenesis, muscle and fat development, as well as contractions of burns, largely depend on mechanical forces and their signals resulting from them. For modelling fundamental processes, like cell differentiation, the determination of mechanical stimuli is crucially important. In many cases, one uses a continuumbased model to simulate cell densities and mechanical forces.

F. J. Vermolen $(\bowtie)$

Delft Institute of Applied Mathematics, Delft University

of Technology, Delft, The Netherlands

e-mail: F.J.Vermolen@tudelft.nl

A. Gefen

Department of Biomedical Engineering, Tel Aviv University, 69978 Tel Aviv, Israel

e-mail: gefen@eng.tau.ac.il
However, in other cases, one rather relies on a cellular treatment where all cells are modelled as individual entities. To compute the mechanical force balance, one often uses a finite-element treatment to compute local displacements and stresses and strains. To compute the local displacements, strains and stresses, the boundary value problem for mechanical equilibrium is often formulated by applying a force boundary condition on the interface between the cell and extracellular matrix. In the case that cells migrate by random walk or by any other signal (being chemotaxis or haptotaxis for instance), one has to solve a moving boundary problem, which complicates the numerical solution schemes largely. In this work, where a cellular approach is used, an alternative approach is proposed, where the cellular forces are incorporated in the mechanical equilibrium directly instead of by the use of boundary conditions. This amounts to solving an elliptic partial differential equation with a moving 'source'-function as a result of cellular forces on the extracellular matrix, whereas all boundary conditions are implemented on the same spatial position at all times. The present approach allows a simplified numerical treatment, and to our awareness, this approach is original. Using a hybrid approach where cells are treated as individual entities, and where, however, mechanical forces are treated by a continuum-based modelling approach, has been presented in studies by Vermolen and Gefen (2013). The innovation of the present paper concerns the mechanical treatment, its application to burns and its subsequent application to generic cyclic loadings, that, for instance, occur in cardiac cycles. The scope of the present paper is the computation of cellular forces through the cell boundaries on the extracellular matrix that surrounds the cells. The treatment is generic and allows extensions to model mechanics-related processes in biology.

Cellular-based modelling approaches are for instance the cellular Potts model where the cell shape and position are 
represented by 'occupying' or 'not occupying' certain control areas that are dictated by a discrete lattice. Examples are presented in Merks and Koolwijk (2009), Graner and Glazier (1992) and van Oers et al. (2014). In the present approach, we allow cells to move continuously over a specified domain. This continuous cellular approach was done in earlier studies by Byrne and Drasdo (2009), Groh and Louis (2010) and Neilson et al. (2011). Cell-cell contacts in this study will be modelled using the principles outlined in Gefen (2010) and Vermolen and Gefen (2012). We believe that our approach of mechanical boundary forces can also be applied in a cellular Potts framework.

The key innovation in this paper, which is the treatment of the mechanical forces exerted by the cell walls on the extracellular matrix, is applied to modelling contraction and contractures of burns. Patients suffering from burn injuries often lose local mobility of body parts, as well as they end up having excessive scar tissue. In the present study, we model the occurrence of a contracture, which is a permanent contraction of a wound. In many modelling studies on wound healing, wound contraction is taken into account; however, contractures are rarely dealt with. In this study, we present a simplified framework for the modelling of contractures, which can be used in more realistic mathematical models to actually predict the likelihood for the occurrence of contractures, as well as the likelihood for the occurrence of excessive scar formation. Mathematical models for wound contraction can be found in the papers by Olsen et al. (1995), Murray (2004) and Vermolen and Javierre (2012) and most recently in Valero et al. (2014), where the last mentioned paper provides a breakthrough in modelling regarding the relation between myofibroblasts and local dilatations. A second application involves modelling cyclic loading where the present model allows to quantify the impact of phase perturbations in the cycles of individual cells on heart performance.

The paper is organised as followed. First, the mathematical model is presented, where we start by considering the most generic framework and gradually introduce various special cases and simplifications. Subsequently, we present the numerical method to solve the problem, and this presentation is followed by description of the results where the model is applied to simulate contractures. Finally, the model is discussed and some conclusions are drawn.

\section{Mathematical model}

The mathematical model roughly consists of two parts. First, we treat the computation of the forces, strains, stresses over the domain of computation as a result of the pulling forces of the cells as well as mechanical consequences due to permanent stresses. Secondly, we consider the migration, death and differentiation of cell types. The latter part is referred to as the biological part.

\subsection{Treatment of the mechanical balance}

We consider an open, bounded domain of computation $\Omega$ with boundary $\partial \Omega$, where we have the following mechanical force balance

$\nabla \cdot \underline{\underline{\sigma}}+\mathbf{F}=\mathbf{0}, \quad$ in $\Omega$,

where $\underline{\sigma}, \mathbf{F}$, respectively, represent the stress tensor and the internal forces. The internal forces result from the cells that exert forces. Regarding stress-strain dependence, in the present paper, we use simple Hooke's Law. The domain of computation, which represents a certain tissue region, is tied to the surrounding tissue by spring forces, and hence, the boundary condition on $\partial \Omega$ is given by

$\underline{\underline{\sigma}} \cdot \mathbf{n}+K \mathbf{u}=\mathbf{0}$

Here, $K \geq 0$ represents a spring force constant. Note that $K \longrightarrow \infty$ implies that $\mathbf{u} \longrightarrow \mathbf{0}$ and that $K \longrightarrow 0$ transfers the boundary into a free boundary. Note that $K=0$ makes the elliptic problem singular, and note that integration over the domain of computation gives

$\int_{\partial \Omega} \mathbf{u d} \Gamma+\frac{1}{K} \int_{\Omega} \mathbf{F} \mathrm{d} \Omega=0$,

as a property for the displacement on the outer boundary.

\subsubsection{Cellular temporary contractile forces}

Imagine that $\mathbf{F}$ represents a point force at the origin directed in the direction $\hat{\mathbf{a}}$, represented by

$\mathbf{F}(\mathbf{x})=\hat{\mathbf{a}} \delta(\mathbf{x})$,

where â represents a unit vector, and $\delta(\mathbf{x})$ represents the Dirac delta distribution, formally defined by

$\left\{\begin{array}{l}\delta(\mathbf{x})=0, \quad \text { if } \mathbf{x} \neq \mathbf{0}, \\ \int_{Q} \delta(\mathbf{x}) \mathrm{d} \Omega=1, \quad \text { if } \mathbf{0} \in Q,\end{array}\right.$

where $Q$ is open. Then, the solution to Eq. (1) gives the displacement field over $\Omega$ as a result of the point source on the origin in the direction of $\hat{\mathbf{a}}$. This principle is used to compute the overall displacement field as a result of forces that are exerted over a line in $\mathbb{R}^{2}$ or over a surface in $\mathbb{R}^{3}$. To this extent, we consider a cell that pulls or pushes on the extracellular matrix around it. The treatment is presented for the two-dimensional case, the reader will be able to extend this principle to three dimensions. We consider a cell, occupying $\Omega_{\mathrm{c}} \subset \Omega$, with boundary $\Gamma_{\mathrm{c}}$, which is a closed curve in $\mathbb{R}^{2}$ and first we treat the case that the cell boundary is polygonal. Let $N$ be the number of line segments of $\Gamma_{\mathrm{c}}$, with midpoints $\mathbf{x}_{k}$, and unit normal vectors $\mathbf{n}\left(\mathbf{x}_{k}\right)$ (pointing into $\Omega_{\mathrm{c}}$ ) with respective lengths $\Delta \Gamma\left(\mathbf{x}_{k}\right)$. Further, let $P\left(t, \mathbf{x}_{k}\right)$ be the magnitude of the force per unit length of $\Gamma_{\mathrm{c}}$ that is exerted at 
position $\mathbf{x}_{k}$. Then, the total force in Eq. (2) is given by

$\mathbf{F}_{N}(\mathbf{x})=\sum_{k=1}^{N} P\left(t, \mathbf{x}_{k}\right) \mathbf{n}\left(\mathbf{x}_{k}\right) \delta\left(\mathbf{x}-\mathbf{x}_{k}\right) \Delta \Gamma_{k}$.

Note that using a limit transition $N \longrightarrow \infty$, i.e. $\Delta \Gamma=$ $\max _{j} \Delta \Gamma_{j} \longrightarrow 0$, gives

$\mathbf{F}_{N}(\mathbf{x}) \longrightarrow \mathbf{F}(\mathbf{x})=\int_{\Gamma_{\mathrm{c}}} P\left(t, \mathbf{x}^{\prime}\right) \mathbf{n}\left(\mathbf{x}^{\prime}\right) \delta\left(\mathbf{x}-\mathbf{x}^{\prime}\right) \mathrm{d} \Gamma^{\prime}$,

as $N \longrightarrow \infty$,

if $\Gamma_{\mathrm{c}}$ is a piecewise, smooth curve. Having a set of $N_{\mathrm{c}}$ cells, with index $j$, allows to apply the principle of superposition due to linearity of the partial differential equations, and hence, we get

$\mathbf{F}(\mathbf{x})=\sum_{j=1}^{N_{\mathrm{c}}} \int_{\Gamma_{\mathrm{c}}^{j}(t)} P\left(t, \mathbf{x}^{\prime}\right) \mathbf{n}\left(\mathbf{x}^{\prime}\right) \delta\left(\mathbf{x}-\mathbf{x}^{\prime}\right) d \Gamma^{\prime}$.

This treatment allows cells to deform and to migrate. We realise that we neglect that the force balance needs a change of sign inside the cell. This neglection is justified if the cells are much smaller than the actual domain of computation. In the two-dimensional cases that we consider in this study, we mechanically treat cells as objects that exert traction in the normal direction of its boundary.

Next, we motivate for one cell that the current formalism is equivalent to actually imposing a natural force boundary condition in the direction of the normal vector on the cell boundary. For this purpose, we introduce the tangential unit vector (in positive direction, which is counterclockwise) on the cell boundary (being a curve in two dimensions) by $\tau$. Since besides the cell traction force on the cell boundary, there are no body-forces, mechanical equilibrium dictates that the divergence of the stress is zero in the domain. Furthermore, on its boundary, the cell exerts a force $\mathbf{F}_{\mathrm{c}}(t, \mathbf{x})$ on time $t$ and position $\mathbf{x}$ on $\Gamma_{\mathrm{c}}$, which is decomposed into the normal and tangential component, using the unit normal and unit tangential vectors, by

$$
\begin{aligned}
\mathbf{F}_{\mathrm{c}}(t, \mathbf{x})= & \left(\mathbf{F}_{\mathrm{c}}(t, \mathbf{x}) \cdot \mathbf{n}(t, \mathbf{x})\right) \mathbf{n}(t, \mathbf{x})+\left(\mathbf{F}_{\mathrm{c}}(t, \mathbf{x})\right. \\
& \cdot \tau(t, \mathbf{x})) \tau(t, \mathbf{x}) .
\end{aligned}
$$

Since we only incorporate forces that are exerted by the cell in the direction of the normal vector with magnitude $P(t, \mathbf{x})$, we use

$\mathbf{F}_{\mathrm{c}}(t, \mathbf{x}) \cdot \mathbf{n}(t, \mathbf{x})=P(t, \mathbf{x}), \quad \mathbf{F}_{\mathrm{c}}(t, \mathbf{x}) \cdot \tau(t, \mathbf{x})=0$.

Herewith, we consider one cell with the following boundary value problem for the displacement $\mathbf{u}$ $\begin{cases}\nabla \cdot \underline{\sigma}=0, & \text { in } \Omega^{\prime}, \\ \underline{\underline{\sigma}}=-\mathbf{F}_{\mathrm{c}}(t, \mathbf{x}), & \text { on } \Gamma_{\mathrm{c}}, \\ \underline{\bar{\sigma}} \mathbf{n}+K \mathbf{u}=\mathbf{0}, & \text { on } \partial \Omega,\end{cases}$

where $\Omega^{\prime}=\Omega \backslash \bar{\Omega}_{\mathrm{c}}$, where $\Omega_{\mathrm{c}}$ represents the open region occupied by the cell. From physical considerations, we are interested in continuous solutions, and we safely take the existence of such a solution for granted. We first evaluate the contribution of the term due to cell traction exerted in the normal direction of the cell boundary to the weak form for one cell only. The extension to multiple cells follows from a superposition argument. This term is given by

$$
\begin{aligned}
& \int_{\Omega} \phi(\mathbf{x}) \cdot \mathbf{F}(t, \mathbf{x}) \mathrm{d} \Omega=\int_{\Omega} \phi(\mathbf{x}) \lim _{N \rightarrow \infty} \sum_{k=1}^{N} P\left(t, \mathbf{x}_{k}\right) \mathbf{n}\left(\mathbf{x}_{k}\right) \\
& \quad \times \delta\left(\mathbf{x}-\mathbf{x}_{k}\right) \Delta \Gamma_{k} \mathrm{~d} \Omega=\lim _{N \rightarrow \infty} \sum_{k=1}^{N} P\left(t, \mathbf{x}_{k}\right) \mathbf{n}\left(\mathbf{x}_{k}\right) \Delta \Gamma_{k} \\
& \quad \times \int_{\Omega} \phi(\mathbf{x}) \delta\left(\mathbf{x}-\mathbf{x}_{k}\right) \mathrm{d} \Omega .
\end{aligned}
$$

The interchange of summation and integration is allowed because of integrability of $\phi \in H^{1}(\Omega)$ and of $P$. Next the fundamental property $\int_{\Omega} f(\mathbf{x}) \delta(\mathbf{x}) \mathrm{d} \Omega=f(0,0)$, if the origin is contained in the open domain $\Omega$, is used to infer that

$$
\int_{\Omega} \phi(\mathbf{x}) \cdot \mathbf{F}(t, \mathbf{x}) \mathrm{d} \Omega=\lim _{N \rightarrow \infty} \sum_{k=1}^{N} P\left(t, \mathbf{x}_{k}\right) \mathbf{n}\left(\mathbf{x}_{k}\right) \cdot \phi\left(\mathbf{x}_{k}\right) \Delta \Gamma_{k} .
$$

Finally, in the above expression, one recognises the definition of a (Riemann) line-integral to finally conclude that

$\int_{\Omega} \phi(\mathbf{x}) \cdot \mathbf{F}(t, \mathbf{x}) \mathrm{d} \Omega=\int_{\Gamma_{\mathrm{c}}} P(t, \mathbf{x}) \mathbf{n} \cdot \phi \mathrm{d} \Gamma$.

Hence, we get for the weak form of the first problem defined by Eqs. $(1,2,7)$ on $\Omega$ :

$$
\left\{\begin{array}{l}
\text { Find } \mathbf{u}_{1} \in V_{1} \times V_{1} \text { such that } \\
\int_{\Omega} \underline{\underline{\sigma}}\left(\mathbf{u}_{1}\right): \underline{\epsilon}(\phi) \mathrm{d} \Omega+\int_{\partial \Omega} K \mathbf{u}_{1} \cdot \phi \mathrm{d} \Gamma \\
=\int_{\Gamma_{\mathrm{c}}} P(\bar{t}, \mathbf{x}) \mathbf{n} \cdot \phi \mathrm{d} \Gamma, \\
\text { for all } \phi \in V_{1} \times V_{1} .
\end{array}\right.
$$

Here, we used the definition of the Dirac delta distribution, and we set $V_{1}:=H^{1}(\Omega) \cap C(\bar{\Omega})$. The weak form for the second problem, defined by on $\Omega^{\prime}$, is

$$
\left\{\begin{array}{l}
\text { Find } \mathbf{u}_{2} \in V_{2} \times V_{2} \text { such that } \\
\int_{\Omega^{\prime}} \underline{\underline{\sigma}}\left(\mathbf{u}_{2}\right): \underline{\underline{\epsilon}}(\phi) \mathrm{d} \Omega+\int_{\partial \Omega} K \mathbf{u}_{2} \cdot \phi \mathrm{d} \Gamma \\
\quad=\int_{\Gamma_{\mathrm{c}}} P(t, \mathbf{x}) \mathbf{n} \cdot \phi \mathrm{d} \Gamma \\
\text { for all } \phi \in V_{2} \times V_{2} .
\end{array}\right.
$$

Here, we define $V_{2}:=H^{1}\left(\Omega^{\prime}\right) \cap C\left(\overline{\Omega^{\prime}}\right)$. The right-hand side of the above equation follows from the boundary condition 
on $\Gamma_{\mathrm{c}}$. Using linearity, the difference between the two weak forms satisfies

$$
\left\{\begin{array}{l}
\text { Find } \mathbf{u}_{2}-\mathbf{u}_{1} \in V_{2} \times V_{2} \text { such that } \\
\int_{\Omega^{\prime}} \underline{\bar{\sigma}}\left(\mathbf{u}_{2}-\mathbf{u}_{1}\right): \underline{\underline{\epsilon}}(\phi) \mathrm{d} \Omega+\int_{\partial \Omega} K\left(\mathbf{u}_{2}-\mathbf{u}_{1}\right) \\
\quad \cdot \phi \overline{\mathrm{d}} \Gamma=-\int_{\Omega_{\mathrm{c}}} \underline{\bar{\sigma}}\left(\mathbf{u}_{1}\right): \underline{\underline{\epsilon}}(\phi) d \Omega \\
\text { for all } \phi \in V_{2} \times V_{2} .
\end{array}\right.
$$

Since $\mathbf{u}_{1} \in V_{1} \times V_{1}$ (we have continuity and hence boundedness), we have that the right-hand side tends to zero as the area of $\Omega_{\mathrm{c}}$ tends to zero. This implies that $\mathbf{u}_{2} \longrightarrow \mathbf{u}_{1}$ as $\operatorname{Area}\left(\Omega_{\mathrm{c}}\right) \longrightarrow 0$. The physical reason for the difference between the two formulations is that the first formulation (i.e. the Dirac Delta Form) incorporates the reaction of the cellular body on the boundary forces that the cells exert on the extracellular matrix.

\subsubsection{Permanent contractile forces}

Once the fibroblasts differentiate into myofibroblasts, they start pulling more firmly on the surrounding tissue. Next to stronger contractile loading, they rearrange the polymeric molecules that form the basis of the collageneous extracellular matrix. The chemical bonds are shortened so that effectively, the myofibroblasts kind of make knots on the polymeric molecules. These knots effectively shorten the polymeric chains such that residual stresses remain. These residual stresses are phenomenologically modelled by treating them in the same manner as external point loading forces. Since the development of this forcing has a evolutionary nature, it takes some time until maximum forcing is reached. Since these forces are residual and can only be reduced during the everlasting remodelling process, they are called the permanent contractile forces, or more shortly, the plastic forces. The resulting permanent deformation of the wound is then referred to as the plastic wound deformation. Furthermore, the resulting reduction in the wound area is called the contracture, whereas physicians only speak of a contracture if the permanent contraction causes physical disabilities to the patient. To model these plastic forces, the wound area is divided into control volumes, which coincide with the elements in the finite-element discretisation that will be presented later. The amount of plastic force in such an element is determined by the amount of time and by the portion of area that has been occupied by myofibroblasts. To this extent, we introduce an effective occupancy time, $\tau_{e}$ for each control volume/element $e$, that satisfies the following evolutionary differential equation:

$\frac{\mathrm{d} \tau_{e}}{\mathrm{~d} t}=\frac{\mathcal{A}\left(\Omega_{\mathrm{c}}(t) \cap \Omega_{e}(t)\right)}{\mathcal{A}\left(\Omega_{e}(t)\right.}$.

Here, $\mathcal{A}($.$) denotes the area, \Omega_{\mathrm{c}}(t)$ and $\Omega_{e}(t)$, respectively, denote the regions occupied by a myofibroblast and a control volume/element. This pseudo-time $\tau_{e}$ is biologically inter- preted as the effective time interval during which a control volume, or an element, has been occupied by myofibroblasts. If the portion of a control volume that has been occupied by myofibroblasts is small, then the increment of $\tau_{e}$ is small related to the physical time interval. The pseudo-time $\tau_{e}$ measures the time interval and impact (through the ratio of areas) as a result of the presence of myofibroblasts in a control volume or element over time. We compute the permanent forcing over the domain of computation by solving

$\frac{\mathrm{d} P_{e}}{\mathrm{~d} \tau_{e}}=\alpha_{\mathrm{p}}\left(P_{\max }-P_{e}\right), P_{e}(0)=0$,

with solution

$P_{e}\left(\tau_{e}\right)=P_{\max }\left(1-e^{-\alpha_{\mathrm{p}} \tau_{e}}\right)$,

where $\alpha_{\mathrm{p}} \in \mathbb{R}$ is kept constant. Here, $P_{\max }$ denotes the maximum forcing due to shortening of the extracellular bonds, and $\alpha_{\mathrm{p}}$ represents the shortening rate of the polymeric chains in the extracellular matrix. The forces are modelled as line forces on the control volume, similarly to the temporary forces but now over control volume boundaries by

$\mathbf{F}_{\mathrm{p}}\left(\tau_{e}, \mathbf{x}\right)=\sum_{l=1}^{N_{e_{l}}} P_{e_{l}}\left(\tau_{e}\right) \int_{\partial \Omega_{e_{l}}(t)} \mathbf{n}\left(\mathbf{x}^{\prime}\right) \delta\left(\mathbf{x}-\mathbf{x}^{\prime}\right) \mathrm{d} \Gamma^{\prime}$.

In the above equation, all contributions from the control volumes have been taken into account by summing over all $N_{e_{l}}$ control volumes.

\subsection{The cellular model}

Regarding the cells, of which we consider fibroblasts and myofibroblasts only, we take cell migration, differentiation and apoptosis into account. In the present model, we assume cells to migrate by

- random walk: cells diffuse over the extracellular matrix, also if there would be no diffusion of cells, cell migration exhibits randomness due to randomness in the structure of the extracellular matrix,

- chemotaxis: cells are attracted to cytokines (VEGF) that are secreted by immune cells, such as macrophages,

- cell-cell contacts: cells are not allowed to overlap and cells will repel each other by contact forces,

- mechanical drag: as a result of local displacements in the extracellular matrix, cells will be dragged over the extracellular matrix. This is also known as passive convection.

Let $X_{j}(t)$ be the position of the centre of cell $j$, then under the assumption of random walk and chemotaxis, cell migration 
is modelled by

$$
\begin{gathered}
\mathrm{d} \mathbf{X}_{j}(t)=\sqrt{2 D} \mathrm{~d} \mathbf{W}(t)+\mathbf{v}_{\mathrm{p}}(t) \mathrm{d} t+\mu \nabla c\left(t, \mathbf{X}_{j}(t)\right) \mathrm{d} t \\
\text { where } t_{j}^{b}<t<t_{j}^{d}, \text { and } \mathbf{X}_{j}\left(t_{j}^{b}\right) \text { is given, }
\end{gathered}
$$

where $D, \mu, c, t_{j}^{b}$ and $t_{j}^{d}$, respectively, denote the macroscale diffusion coefficient of the cell type, chemotactic sensitivity, concentration of the chemical agent to which the cell is attracted $(\mu>0)$ or repelled $(\mu<0)$, time of birth and death of cell $j$. We take $t_{j}^{b}=0$ for all $j$. The times of death are determined by the stochastic processes for cell death (satisfying a Poisson process). The vector $\mathbf{v}_{\mathrm{p}}(t)$ denotes the migration speed of the cell as a result of summing over passive convection $\left(\mathbf{v}_{\mathrm{d}}(t)\right)$ and cell-cell contact forces $\left(\mathbf{v}_{\mathrm{c}}(t)\right)$, that is

$\mathbf{v}_{\mathrm{p}}(t)=\mathbf{v}_{\mathrm{c}}(t)+\mathbf{v}_{\mathrm{d}}(t)$.

Further, W denotes a vector Wiener process. Wiener processes or Brownian motion is described in more detail in Steele (2001). The contact forces have been described in Vermolen and Gefen (2012). Mechanical drag is incorporated by

$\mathbf{v}_{\mathrm{d}}(t)=\frac{\mathrm{d} \mathbf{u}}{\mathrm{d} t}$,

where $\mathbf{u}$ denotes the displacement vector.

Subsequently, cell differentiation from a fibroblast to a myofibroblast has been incorporated as a stochastic process. It is well-known that differentiation of fibroblasts to myofibroblasts takes place as a result of expansion forces (Valero et al. 2014) of the wound and as a result of a high levels of VEGF. Therewith, this differentiation predominantly takes place near the edge of the wound. Since the current paper only focuses on modelling cell-based and control volumebased contractile forces, we incorporate this by postulating that the probability of differentiation is maximal on the rim of the wound. To make this slightly easier, we consider the following probability behaviour, $\mathcal{P}_{\mathrm{D}}$ of the differentiation process

$\mathcal{P}_{\mathrm{D}}=\mathcal{P}_{\mathrm{D}}^{0} \exp \left(-\alpha_{\mathrm{D}}\left(\left\|\mathbf{X}_{i}\right\|-R\right)^{2}\right)$,

where $R$ denotes the distance from the wound centre where the likelihood for differentiation is maximal. Further, $\mathcal{P}_{\mathrm{D}}$ denotes the probability of differentiation per unit of time. This approach is very phenomenological since one could better use a mechanical signal such as the positive part of the dilatation as a trigger for the differentiation rate. This treatment, however, does not fall in the scope of the paper, which is merely descriptive on the treatment of point sources and plastic forces. In fact, it is debatable whether such a purely stochastic approach is right at all from a biological point of view.
Further, cell death is treated as a stochastic process as well. The probability of death for myofibroblasts is assumed to be ten times as large (apoptosis) as for fibroblasts in some of our simulations.

\section{Numerical method}

We explain the finite-element method for the approximation of the solution of the elasticity equations and the solution of the stochastic differential equations, as well as the stochastic processes for cell differentiation and apoptosis in two separate subsections.

\subsection{Finite-element method for mechanical balance}

The finite-element treatment of most of the terms in the above equation is standard; however, the treatment of the right-hand side of Eq. (15) will be elaborated in some more detail. To this extent, we split the integral into the contributions resulting from the two coordinate directions. Though the extension to three dimensions can be made easily, the formalism is only presented in two dimensions. Considering the treatment of the weak form in the direction of the $x$-coordinate, one obtains in the weak formulation (for one cell) when multiplied by the $i$ th basis function

$$
\begin{aligned}
\int_{\Omega} \phi_{i}(\mathbf{x}) F^{x}(\mathbf{x}) \mathrm{d} \Omega= & \int_{\Omega} \phi_{i}(\mathbf{x}) \int_{\Gamma_{\mathrm{c}}} P\left(t, \mathbf{x}^{\prime}\right) n_{x}\left(\mathbf{x}^{\prime}\right) \\
& \times \delta\left(\mathbf{x}-\mathbf{x}^{\prime}\right) \mathrm{d} \Gamma^{\prime} \mathrm{d} \Omega \\
= & \int_{\Gamma_{\mathrm{c}}} \int_{\Omega} \phi_{i}(\mathbf{x}) P\left(t, \mathbf{x}^{\prime}\right) n_{x}\left(\mathbf{x}^{\prime}\right) \\
& \times \delta\left(\mathbf{x}-\mathbf{x}^{\prime}\right) \mathrm{d} \Omega \mathrm{d} \Gamma^{\prime} \\
= & \int_{\Gamma_{\mathrm{c}}} \phi_{i}\left(\mathbf{x}^{\prime}\right) P\left(t, \mathbf{x}^{\prime}\right) n_{x}\left(\mathbf{x}^{\prime}\right) \mathrm{d} \Gamma^{\prime} .
\end{aligned}
$$

Since $\phi_{i} \in H^{1}(\Omega)$, we were allowed to interchange the order of the integration in the above formula. The above equation only gives a contribution to $i$ if the basis function $\phi_{i} \neq 0$ for some $\mathbf{x}^{\prime} \in \Gamma_{\mathrm{c}}$. In case of a circular cell, a polygonal integration of the cell boundary makes sense. To simplify matters and to save computation time, we approximate the cell boundary by a square and integrate over the cell boundary with sides $h=\sqrt{\pi} R_{\mathrm{c}}$, where $R_{\mathrm{c}}$ is the cell radius, to this extent the above equation is approximated by

$$
\int_{\Omega} \phi_{i}(\mathbf{x}) F^{x}(\mathbf{x}) \mathrm{d} \Omega=\left(\phi_{i}\left(\mathbf{x}_{1}\right) P\left(t, \mathbf{x}_{1}\right)-\phi_{i}\left(\mathbf{x}_{3}\right) P\left(t, \mathbf{x}_{3}\right)\right) h,
$$

where $\mathbf{x}_{1}=\left(x_{\mathrm{c}}+h / 2, y_{\mathrm{c}}\right), \mathbf{x}_{2}=\left(x_{r m c}, y_{\mathrm{c}}+h / 2\right), \mathbf{x}_{3}=$ $\left(x_{\mathrm{c}}-h / 2, y_{\mathrm{c}}\right)$, and $\mathbf{x}_{2}=\left(x_{\mathrm{c}}, y_{\mathrm{c}}-h / 2\right)$, and here $\left(x_{\mathrm{c}}, y_{\mathrm{c}}\right)$ denote the position of the cell centre. 
We see that the finite-element method needs function evaluations on the midpoints of the cell boundary elements. Since the cells are mobile, we also need a mapping onto the discretisation mesh. Although this can be done, it complicates matters and we need to find which control volume or area a cell is in. This is avoided by a regularised version of the Dirac delta distribution. To this extent, we introduce

$\delta_{\epsilon}(\mathbf{x})=\frac{1}{2 \pi \epsilon^{2}} e^{-\frac{\|\mathbf{x}\|^{2}}{2 \epsilon^{2}}}$.

This expression, which represents the normal distribution, satisfies

$\int_{\mathbb{R}^{2}} \delta_{\epsilon}(\mathbf{x}) \mathrm{d} \Omega=1$,

and it converges to the Dirac delta distribution as $\epsilon \longrightarrow 0$. Let the function $\mathbf{F}$ be given by $\mathbf{F}_{\epsilon}$, then, to this extent, we introduce

$$
\begin{gathered}
\mathbf{F}_{\epsilon}(\mathbf{x})=\int_{\Gamma_{\mathrm{c}}} P\left(t, \mathbf{x}^{\prime}\right) \mathbf{n}\left(\mathbf{x}^{\prime}\right) \delta_{\epsilon}\left(\mathbf{x}-\mathbf{x}^{\prime}\right) \mathrm{d} \Gamma^{\prime} \longrightarrow \mathbf{F}(\mathbf{x}), \\
\text { as } \epsilon \longrightarrow 0 .
\end{gathered}
$$

Hence, in the weak form, we use

$$
\begin{aligned}
\int_{\Omega} \phi_{i}(\mathbf{x}) F_{\epsilon}^{x}(\mathbf{x}) \mathrm{d} \Omega= & \int_{\Gamma_{\mathrm{c}}} \int_{\Omega} \phi_{i}(\mathbf{x}) P\left(t, \mathbf{x}^{\prime}\right) n_{x}\left(\mathbf{x}^{\prime}\right) \\
& \times \delta_{\epsilon}\left(\mathbf{x}-\mathbf{x}^{\prime}\right) \mathrm{d} \Omega \mathrm{d} \Gamma^{\prime} .
\end{aligned}
$$

Taking $P=P_{0}$, and treating the cell boundary as a square like earlier, and applying Newton-Cotes numerical integration over an element, we arrive at the following element vector entries for element $e_{l}$ :

$$
\begin{aligned}
F_{i}^{x}= & \int_{e_{l}} \phi_{i} F^{x}(\mathbf{x}) \mathrm{d} \Omega=\frac{\left|\Delta_{l}\right|}{6} P_{0}\left(\delta_{\epsilon}\left(\mathbf{x}_{\mathbf{i}}-\mathbf{x}_{E}\right)\right. \\
& \left.-\delta_{\epsilon}\left(\mathbf{x}_{\mathbf{i}}-\mathbf{x}_{W}\right)\right) .
\end{aligned}
$$

\subsection{Solution method for stochastic processes}

The stochastic events like cell differentiation and cell death are treated using a randomiser and testing whether the stochastic variable $x i$ satisfies

$\xi:\left\{\begin{array}{l}\leq \mathcal{P} \mathrm{d} t, \text { then event takes place, } \\ >\mathcal{P} \mathrm{d} t, \text { then event does not take place. }\end{array}\right.$

Cell migration is treated by a Euler-Maruyama method, where the deterministic terms are treated at the previous timestep, resulting into

$\mathbf{X}_{j}^{l+1}=\mathbf{X}_{j}^{l}+\mathbf{v}_{\mathrm{p}}^{l} \Delta t+\mu \nabla c\left(t^{l}, \mathbf{X}_{j}^{l}\right) \Delta t+\sqrt{2 D} \Delta t \mathcal{N}$,

where $\mathcal{N} \propto[N(0,1) N(0,1)]^{T}, \Delta t$ denotes the time-step. Further, $l$ denotes the time index.

\section{Numerical simulations}

We consider some simulations for a contracting wound as well as for cardiac contraction cycles. The size of the domain is $1 \mathrm{~mm}$ by $1 \mathrm{~mm}$. For both simulations, we use a structured mesh of $40 \times 40$, which gives mesh sizes of $0.025 \mathrm{~mm}$, which is half the cell radius (see Table 1). Mesh refinement hardly gave any noticeable changes in the numerical solution.

\subsection{Wound contraction}

We illustrate the method by considering an initially square burn wound. The input parameters have been listed in Table 1.

We assume that in the vicinity around the wound, there is an abundance of fibroblasts. We consider the situation that immediately follows after the immune response reaction. There is a concentration profile of VEGF that has a maximum at the centre of the wound. Further, the sources (white blood cells) that secrete the VEGF die away and hence the VEGF levels drop in the course of time as a result of diffusion deeper into the tissue and by chemical reactions. To this extent, we model the concentration profile phenomenologically by

$c(t, \mathbf{x})=\exp \left(-\left(\alpha_{\mathrm{c}} t+\frac{\|\mathbf{x}\|^{2}}{2 D_{\mathrm{c}}}\right)\right)$.

Here, it is easy to take the gradient to account for chemotaxis. Furthermore, the fibroblasts and myofibroblasts are assumed to exert the same contractile forces. Within the wound near its centre, the forces are higher due to a higher amount of VEGF. Regarding the contractile forces exerted by the fibroblasts, we assume these forces to be high in the region around the wound centre whereas values drop away from the wound centre. In the simulations, we use the following qualitative relation:

$P(t, \mathbf{x})= \begin{cases}\hat{P}, & \text { if }\|\mathbf{x}\| \leq R, \\ \tilde{P}, & \text { if }\|\mathbf{x}\|>R .\end{cases}$

In Fig. 1, the wound area as well as the fibroblasts and myofibroblasts are shown. The red region represents the wound region. The circular and diamond regions, respectively, represent the fibroblasts and myofibroblasts. Proliferation of fibroblasts is disregarded here. It can be seen in the early stages, see Fig. 1, that first fibroblasts are surrounding the wound site. Subsequently, they move into the wound and start differentiating to myofibroblasts. In the very early stages, the wound expands a little as a result of the many fibroblasts outside the wound that are pulling. This is believed to trigger differentiation to myofibroblasts, see for instance Valero et al. (2014). We did not take this triggering mechanism into account in the present model. Subsequently, the contraction builds up and becomes substantial. At the later stages, see Fig. 2, first the number of fibroblasts in the wound 
Table 1 Basis values for the various parameters used in the simulations

\begin{tabular}{llll}
\hline Parameter & Meaning & Value & Unit \\
\hline$R_{c}$ & Cell radius & 0.05 & $\mathrm{~mm}$ \\
$N$ & Number of points on cell boundary & 8 & - \\
$\mathcal{P}_{\text {death }}^{\text {fib }}$ & Death rate of fibroblasts & 0.02 & $\mathrm{~h}^{-1}$ \\
$\mathcal{P}_{\text {death }}^{\text {myo }}$ & Death rate of myofibroblasts & 0.02 & $\mathrm{~h}^{-1}$ \\
$\mathcal{P}_{\mathrm{D}}^{0}$ & Differentiation rate & 0.3 & $\mathrm{~h}^{-1}$ \\
$P_{\text {max }}$ & Maximum Plastic Traction density & 5 & $\mathrm{~kg} / \mathrm{mm} / \mathrm{h}^{2}$ \\
$\hat{P}$ & Traction Force by (myo)fibroblasts (wound centre) & 30 & $\mathrm{~kg} / \mathrm{mm}^{2}$ \\
$\tilde{P}$ & Traction Force by (myo)fibroblasts (wound edge) & 15 & $\mathrm{~kg} / \mathrm{mm}^{2} / \mathrm{h}^{2}$ \\
$\alpha$ & Cell mobility & 1 & $\mathrm{~h}^{-1}$ \\
$R$ & Distance from the wound centre & 0.3 & $\mathrm{~mm}$ \\
$D$ & Cell diffusivity & $2 \times 10^{-4}$ & $\mathrm{~mm} / \mathrm{h}$ \\
$\alpha_{\mathrm{c}}$ & Deacy rate of VEGF & 1 & $\mathrm{~h}^{-1}$ \\
$\epsilon$ & Mollifier for delta-function & 0.01 & - \\
$\mu$ & Chemotactic migration coefficient & 1 & $\frac{\mathrm{mm}}{\mathrm{mol}^{3}}$ \\
$\alpha_{\mathrm{D}}$ & Attenuation coefficient of differentiation rate & 2.8 & $\mathrm{~mm}{ }^{-2}$ \\
$\alpha_{\mathrm{p}}$ & Shortening rate of polymers & 1 & $\mathrm{~h}^{-1}$ \\
$E$ & Stiffness modulus & 0.5 & $\mathrm{~kg} / \mathrm{mm}^{2} / \mathrm{h}^{2}$ \\
$v$ & Poisson ratio & 0.35 & - \\
$K$ & Boundary spring forcing & 1 & $\mathrm{~kg} / \mathrm{mm}^{2} / \mathrm{h}^{2}$ \\
$D_{\mathrm{c}}$ & Diffusivity of VEGF & 1 & $\mathrm{~mm}{ }^{2} / \mathrm{h}$ \\
\hline & & &
\end{tabular}

goes down as a result of differentiation to myofibroblasts as well as by cell death. Subsequently, the total number of cells decreases and in the present formulation where no cell proliferation has been taken into account deliberately, the number of cells, even myofibroblasts, goes down to zero in the course of time. The amount of contraction decreases as well during these stages; however, a permanent contraction appears. The total number of cells has been assumed to decrease to zero deliberately to demonstrate how a permanent contraction appears. In reality, a certain number of fibroblasts would be present both inside and outside the wound, which would balance the forces and hence the same pattern would result since the internal and external forces due to fibroblasts would cancel out as a result of the equilibrium behaviour. We note that in reality, the magnitude of pulling forces by fibroblasts would also become the same inside and outside the wound.

To illustrate the amount of contraction, we plot the wound area as a function of time. We consider three cases: no myofibroblast differentiation, moderate myofibroblast differentiation and much myofibroblast differentiation, see Fig. 3. It can be seen that if there is no differentiation of fibroblasts to myofibroblasts, then the wound area tends to its original size after contraction. This happens because there do not appear any myofibroblasts which is the only cell type that is responsible for the plastic forces. If the myofibroblasts are allowed to differentiate to myofibroblasts, then the amount of contraction increases, as well as there is a residual contraction, which is a contracture. The wound area no longer increases up to its original size. This behaviour gets worse as the amount of myofibroblasts increases. All data used to generate Figs. 1 and 2 are taken from Table 1. Another interesting phenomenon is that if there are just few, immobile, myofibroblasts, then the maximum amount of contraction becomes larger. This phenomenon is explained by the ability of the many fibroblasts that can penetrate deep into the wound towards the wound centre. Near the wound centre, it is assumed that, due to the presence of a large concentration of VEGF, the cells being either fibroblasts or myofibroblasts pull harder. If differentiation proceeds at higher levels, then more myofibroblasts will be located near the wound edge, where there they will pull less hard on the surrounding tissue. We, however, realise that these results just illustrate certain trends and that if one really wants to make some statistically sound predictions, then a large number of runs should be considered.

The fact that a final contracture results for excessive differentiation is an undesired effect from clinical point of view.

Next, we consider the impact of the myofibroblast apoptosis rate. In Fig. 4, it can be seen that for higher death rates of myofibroblasts, the degree of contracture decreases and even vanishes if all myofibroblasts would die immediately. Another interesting side effect is that the maximum degree of contraction also decreases. This decrease is a con- 

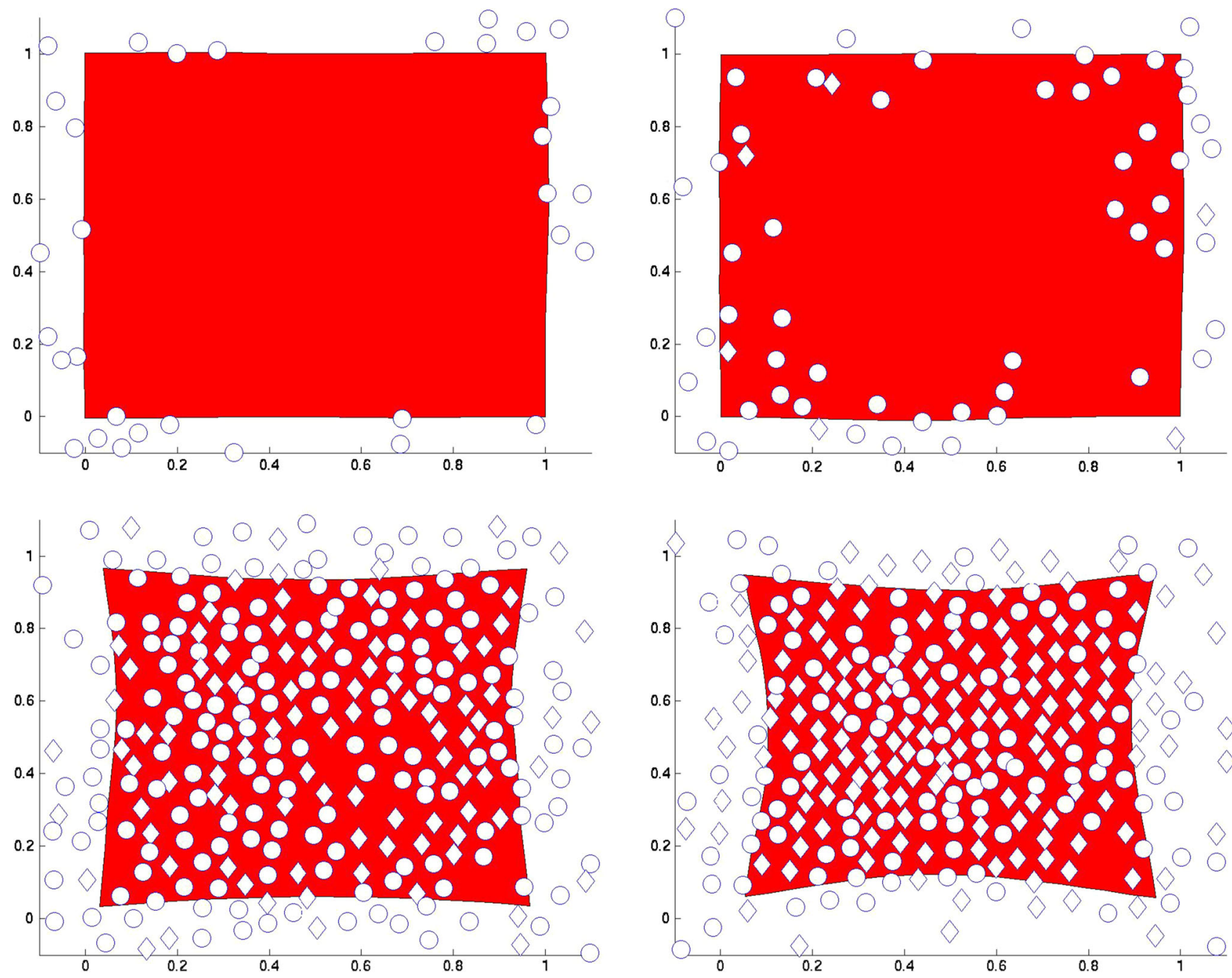

Fig. 1 Snapshots of the contracting wound (red) with fibroblasts (white circles) and myofibroblasts (white diamonds). Times are at $0.1,1,6.5$ and 10h. Cell radius is $0.05 \mathrm{~mm}$

sequence of the fact that many fibroblasts will differentiate into myofibroblasts in the vicinity of the wound edge and hence they will not be able to reach the centre of the wound. The model uses the assumption that the fibroblasts pull harder near the centre of the wound, that is within a circle of radius $R=0.3 \mathrm{~mm}$. The biological justification of the increase in pulling forces follows from the elevated VEGF concentration in the centre of the wound. Lowering the differentiation rate will reduce this effect. The results have not been plotted in the present article.

In Fig. 5, we show the impact of the increase in pulling forces of the myofibroblasts. It can be seen that the maximum degree of contraction increases with increasing myofibroblasts pulling; however, the degree of final contracture hardly increases. Note that the pulling forces by myofibroblasts immediately disappear after death of the myofibroblasts. The results confirm the hypothesis that the myofibroblastinduced decrease in bond lengths is the driving factor for the development of the permanent contraction (i.e. the contracture).

Enlarging of the region, where pulling forces are enlarged for both the fibroblasts and myofibroblasts, gives a higher maximum contraction, but no significant final contracture and maximum degree of contraction, although a small decrease in the level of contracture can be seen. This can be observed from Fig. 6.

\subsection{Loading cycles}

To illustrate the applicability of the model for cell traction forces a bit more, we add some preliminary results for loading cycles. The model that we present here is much too simple for the modelling of myocardial cycles since the present formalism does not accommodate for the effects of cell stress or strain on the contractile force. Furthermore in real muscles, including the heart muscle, muscle cells are directly con- 

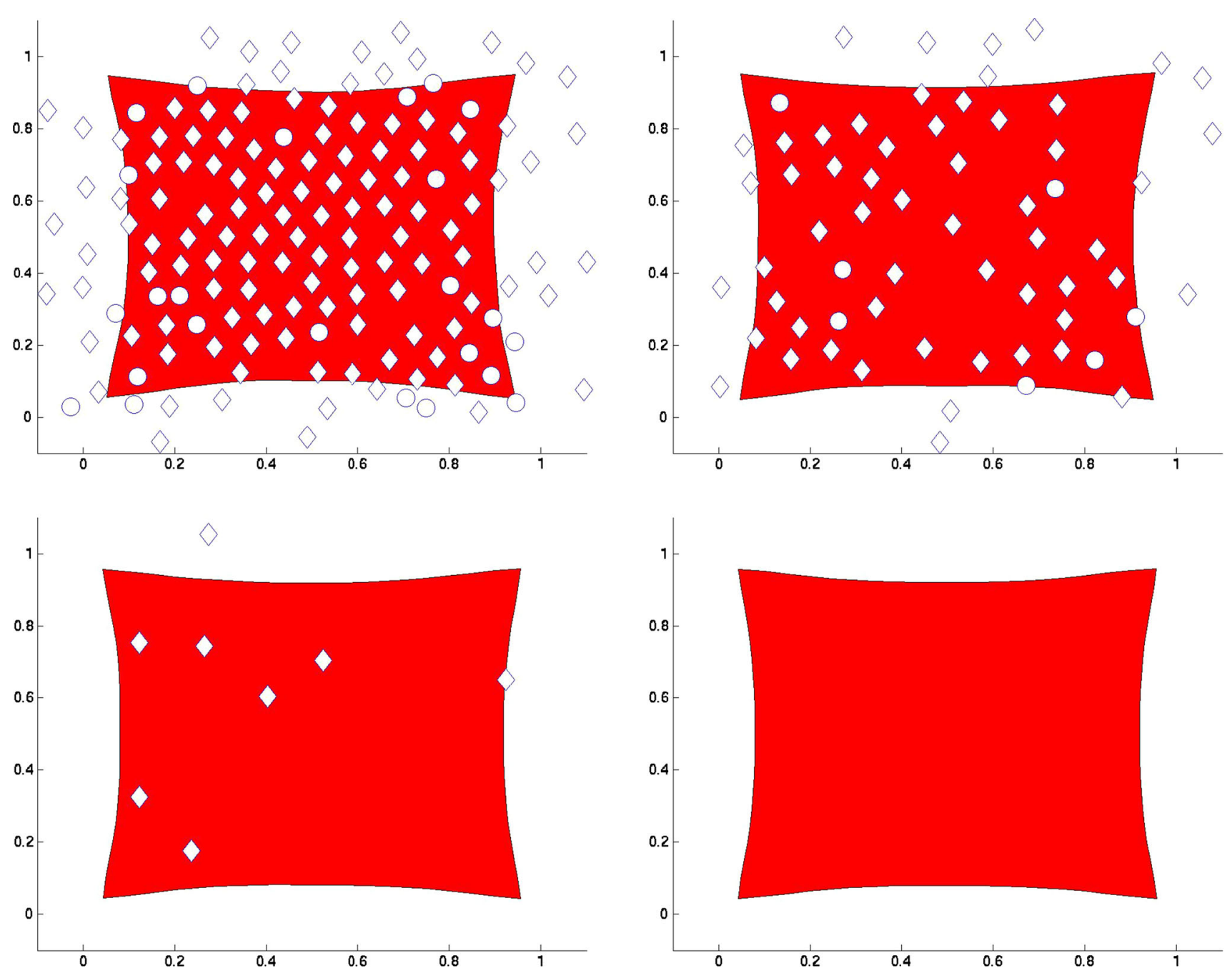

Fig. 2 Snapshots of the contracting wound (red) with fibroblasts (white circles) and myofibroblasts (white diamonds). Times are at 20, 30, 50 and $100 \mathrm{~h}$. The snapshots have been taken from the same run as in Fig. 1. Cell radius is $0.05 \mathrm{~mm}$

nected to one another in large strings, which we do not yet consider in the present model. The cells are assumed to represent muscle cells (myocytes), which are non-mobile except for passive convection, a small amount of random walk and cell-cell contacts. The cells exert a periodical force, which we simplify to

$P_{j}(t)=P^{0} \cos ^{2}\left(2 \pi \omega_{j}(t) t+\phi_{j}(t)\right)$,

where $\omega_{j}$ and $\phi_{j}$, respectively, represent the contraction frequency and phase of the individual cell with index $j$. Cell death and differentiation have been disregarded in these simulations. Some snapshots have been shown in Fig. 7, where it can be seen that the tissue contracts and subsequently expands to its original configuration. No plasticity effects are taken into account. In Fig. 7, the cells were 'seeded' on a circle initially and contraction drags the cells towards the centre. As soon as expansion takes place, then the cells are dragged away from the centre. This cycle repeats.
In order to simulate cyclic defects, we allow the frequency and phase to vary in time and we enlarge the number of cells. For the sake of illustration, we consider a stochastic Wiener process:

$\mathrm{d} \omega_{j}(t)=\sigma_{\omega} \mathrm{d} W(t), \quad \mathrm{d} \phi_{j}(t)=\sigma_{\phi} \mathrm{d} W(t)$,

where $\omega_{j}(0)=1 \mathrm{~s}^{-1}$ and $\phi_{j}(0)=0$. This configuration takes the initial phases and frequencies for all cardiomyocites equal and in the course of time gradual, randomised distortions come into play. In Fig. 8, we present the cycles for several circumstances. First (I) $\sigma_{\omega}=\sigma_{\phi}=0$ (no defects), then (II) $\sigma_{\omega}=0, \sigma_{\phi}=0.5$, (III) $\sigma_{\omega}=0.05, \sigma_{\phi}=0$ and finally (IV) $\sigma_{\omega}=0.05, \sigma_{\phi}=0.5$. It can be seen that if both $\sigma$-values are zero, then the amplitude is large, whereas the amplitude exhibits a very irregular behaviour for the perturbed amplitudes and frequencies, as well as that the average heart area decreases due to the law of large numbers. This can be proved mathematically, which we omit in this paper. For 


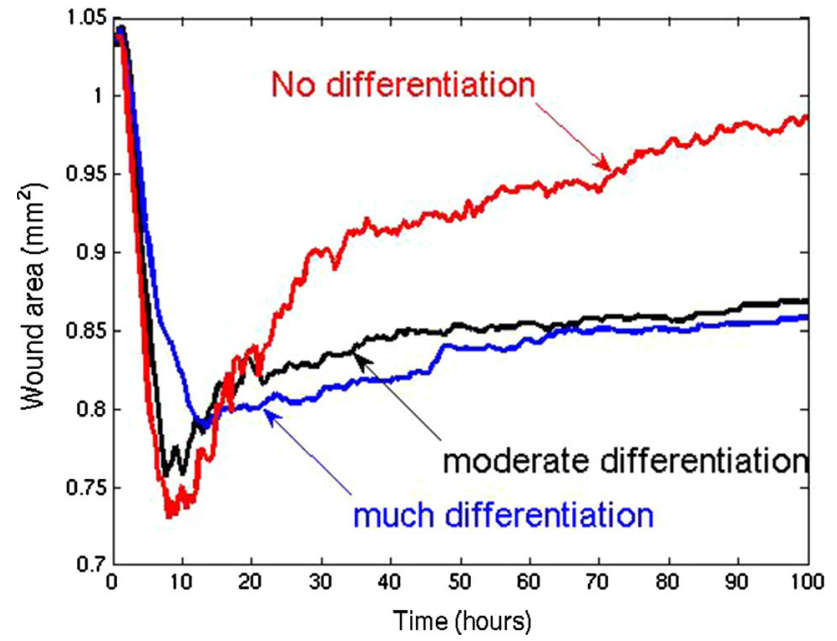

Fig. 3 The wound area as a function of time for different levels of fibroblast to myofibroblast differentiation. The differentiation probabilities per unit of time are given by $\mathcal{P}_{\mathrm{D}}^{0}=0,0.15,0.3 \mathrm{~h}^{-1}$, for, respectively, no differentiation, moderate differentiation and excessive differentiation

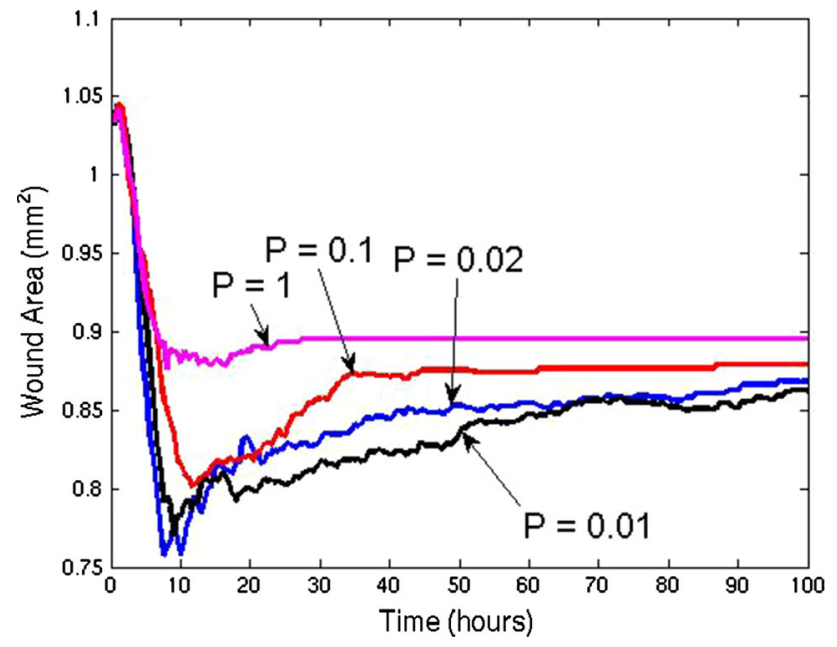

Fig. 4 The wound area as a function of time for different levels of myofibroblast death rate, given by $\mathcal{P}_{\text {death }}^{\text {myo }}=0.01,0.02,0.1,1 \mathrm{~h}^{-1}$, for $\mathcal{P}_{\mathrm{D}}^{0}=0.3 \mathrm{~h}^{-1}$ (excessive differentiation)

these distorted cases, it seems that the heart area would be measured to be larger. The relaxating behaviour of the minima in the undistorted case is only a consequence of sampling issues.

Subsequently, we show the influence of a sudden breakdown, that is sudden death of a considerable portion of the cells, which could occur during a myocardial infarction, in Fig. 9, where the sudden breakdown is modelled by a sudden transition where cells die at a high death rate. The death rate is given by $\mathcal{P}_{\mathrm{D}}=0.25 \mathrm{~s}^{-1}$. This death rate is imposed after $4 \mathrm{~s}$ in the simulation runs. We show the area of the tissue over time, as well as the number of viable cells. It can be seen that the amplitude decreases during the dying process of cells in

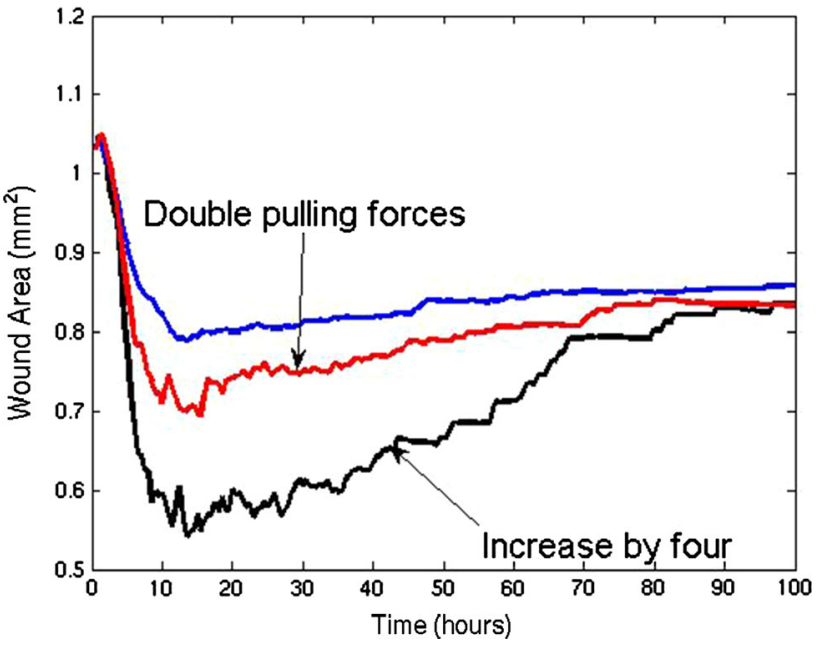

Fig. 5 The wound area as a function of time for different levels of elevated myofibroblasts pulling forces. Results are shown for no elevation of myofibroblast pulling forces, double pulling forces by myofibroblasts and myofibroblast pulling forces that are four times as large as for fibroblasts

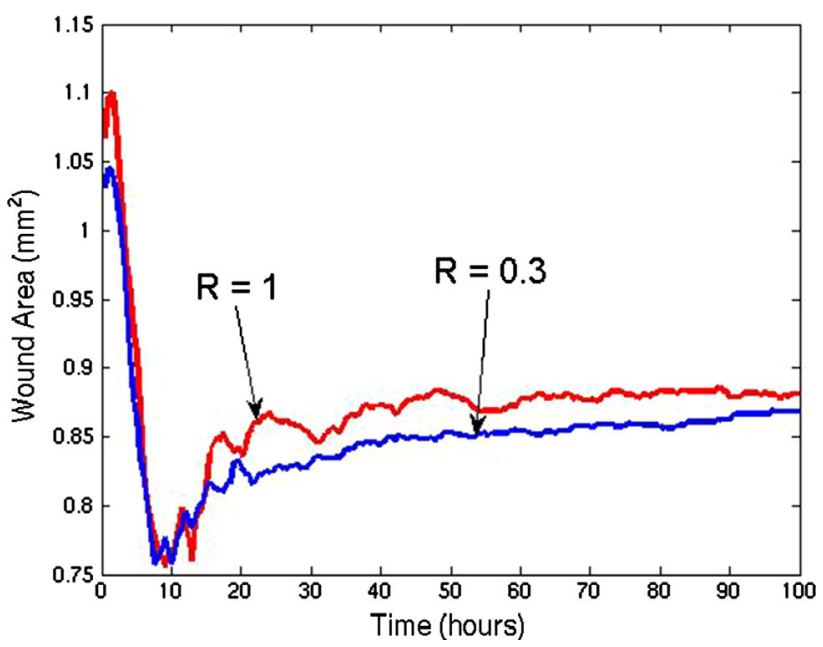

Fig. 6 The wound area as a function of time for different areas where contraction forces are high $R=0.3$ versus $R=1$ at a differentiation rate of $0.15 h^{-1}$

the case of no rhythmic anomalies. Upon distortions on the loading cycle, case (IV), we see that the amplitude decreases due to the differences in frequency and phases, but decreases even further after the sudden breakdown. Another interesting phenomenon is that the tissue area grows towards the fully undistorted case after the sudden breakdown. However, the amplitude decreases as well, which would reduce the blood flow of the patient in the case of a myocardiac infarction, which causes all other negative side effects. Of course, we realise that only a small section is considered in our formalism and that heart attacks often involve a localised region of death of cardiomyocites. 

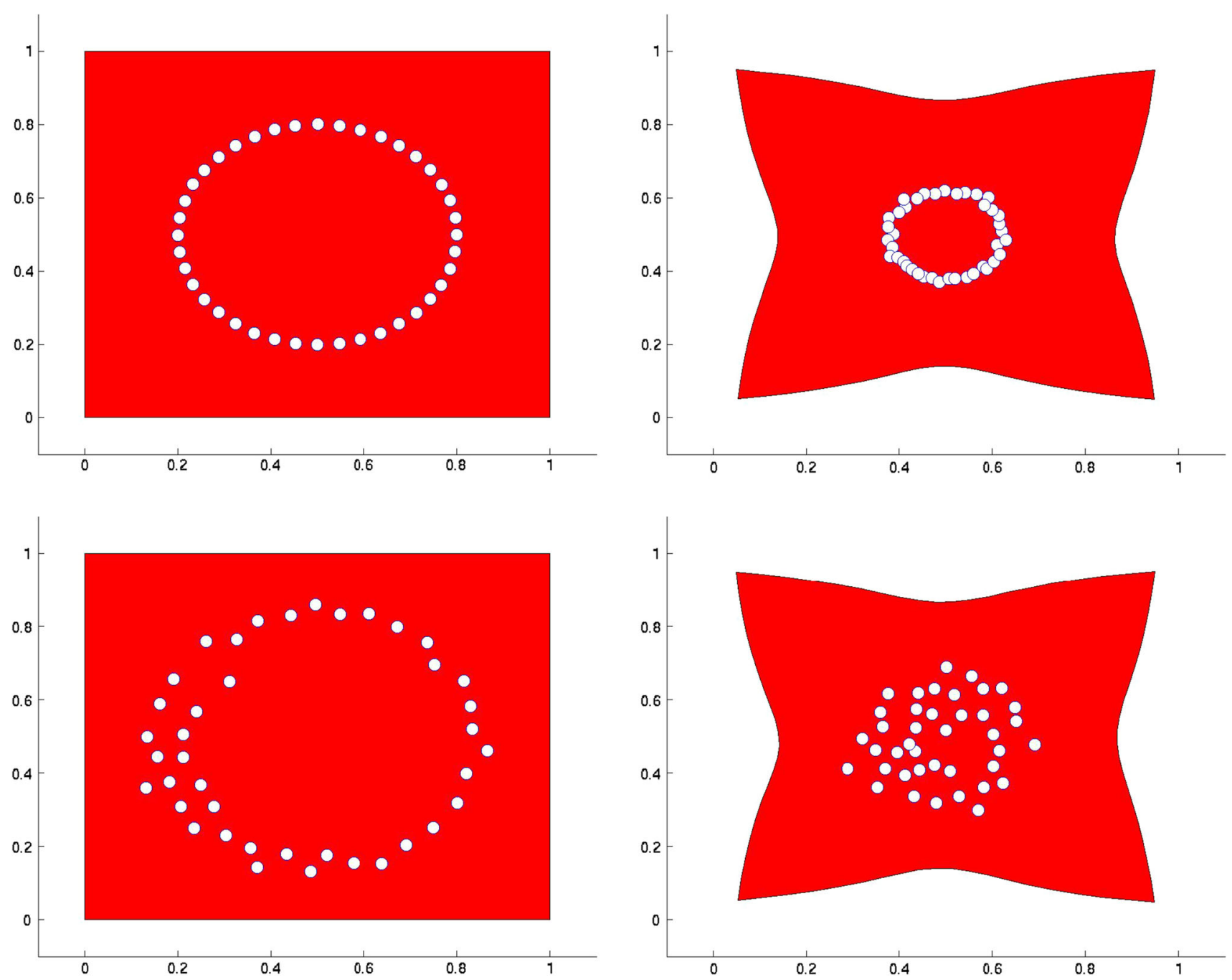

Fig. 7 Snapshots of the contracting 'heart' at consecutive times $(0,0.5,1,1.5) \mathrm{s}$. The red region represents the 'heart', and the white circles represent the cells that exert the cyclic loading. Cell radius is $0.05 \mathrm{~mm}$

Although the modelling of cyclic loadings is very elementary at this stage, the modelling work does have some potential for use in a context of myocardic cycles if the cells are connected as long chains to one another, and if the influence of the extracellular matrix on the cells is incorporated. In one of our current studies, we are working on a modelling set-up where muscle cells are interconnected and form large strings. The potential is that for each cell, we can assign a certain periodic loading with an individual perturbation of the phase in loading so that the impact of rhythmic irregularities over cells can be modelled. With an improved modelling framework for cardiac cycles, using interconnected cardiomyocites and the bidirectional influence between the extracellular matrix and cardiomyocites, one could possibly quantify the influence of rhythmic perturbations of cells onto the irregularity heartbeat (such as atrial fibrillation).

\section{Discussion}

A first point is the stochastic nature of the model, which makes each run represent a single experiment. In order to issue claims concerning comparative results, one has to consider a sound statistical analysis of the intervals of confidence. This statistical analysis has been omitted in the present study and the reason is that the variations of the runs for identical parameters are relatively small, as can be seen in Fig. 10.

The model that has been presented is capable of dealing with temporary and permanent issues in the process of wound contraction. The permanent contraction, that is the contracture, is modelled based on the observation that myofibroblasts shorten the polymeric chains in the extracellular matrix. Apart from these plastic stresses modelled in this way, the mechanical model is based on linear elasticity which is based 

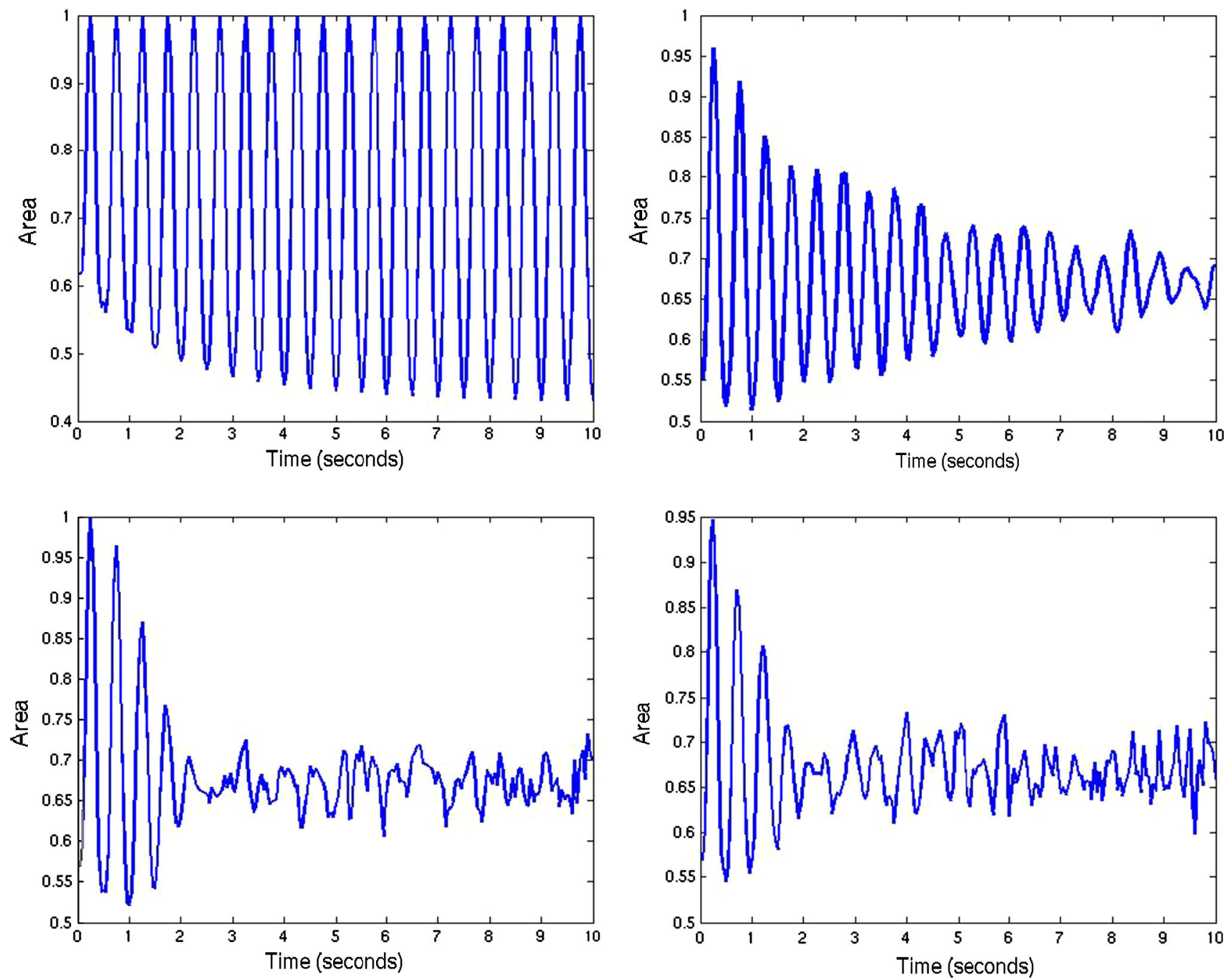

Fig. 8 The area of the contracting 'heart' versus time. From left to right and from top to bottom: (I) $\sigma_{\omega}=\sigma_{\phi}=0$, (II) $\sigma_{\omega}=0, \sigma_{\phi}=0.5$, (III) $\sigma_{\omega}=0.05, \sigma_{\phi}=0$ and finally (IV) $\sigma_{\omega}=0.05, \sigma_{\phi}=0.5$

on infinitesimal strain theory. For some of the resulting contractures, the strains are actually significant, which means that the hypothesis of infinitesimal strain theory is no longer accurate. This issue has not been dealt with properly in the present paper, because the main goal of the present manuscript is the formulation of cellular (pulling) forces on the extracellular matrix, as well as the introduction of residual stresses due to shortening of polymeric chains in the extracellular matrix. The model is applied to wound contraction, but the range of application is much wider. One can also think of modelling obesity or the contraction of muscle cells (myocites or cardiomyocites). The latter case has been illustrated shortly in the manuscript.

One should also realise that the real modelling of contractions and contractures should involve the complex interplay between chemokines and fibroblast-experienced strains and stresses. These issues have been simplified by phenomenological modelling. We are, however, working on a neater framework for the modelling of wound contraction, where the principles that have been developed in this paper are applied.

In two of our modelling studies, we formulated models for cell migration and deformation where only the cell boundary is tracked through its division into nodal points, which are interconnected by springs and connected by springs to the nucleus to model cell stiffness, see for instance Vermolen and Gefen (2012). Although the scope of the present paper is the calculation of cellular forces that are exerted on the extracellular matrix surrounding the cells for a cell-based model where cells are presently treated as equishaped objects, an extension to varying cell shapes is straightforward and is of current research. The work of van Oers et al. (2014) provides an interesting cellular Potts framework where mechan- 

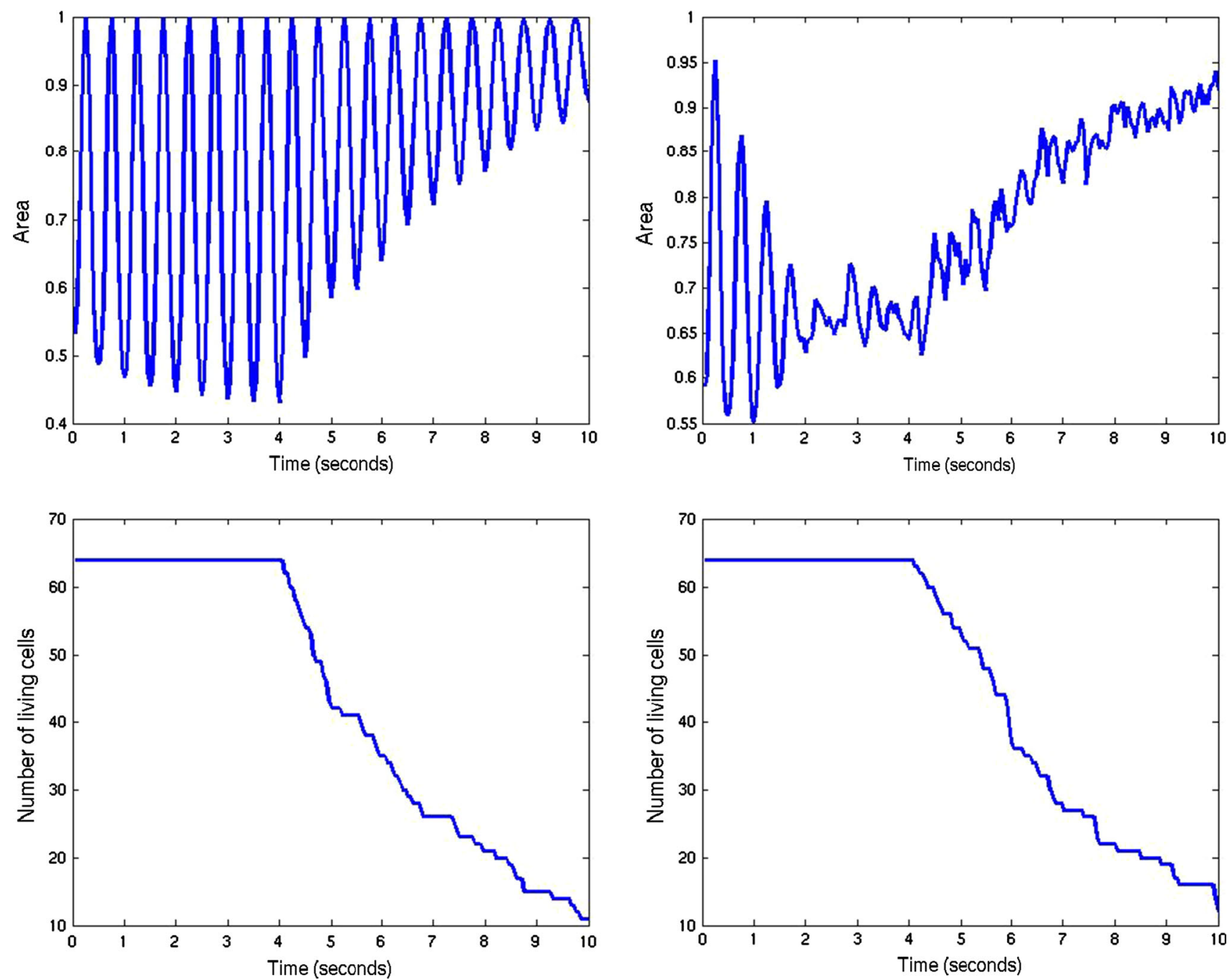

Fig. 9 Top The area of the contracting 'heart' versus time. From left to right case (I) and case (IV); Bottom The number of viable cells after the initiation of the infarction. From left to right case (I) and case (IV)

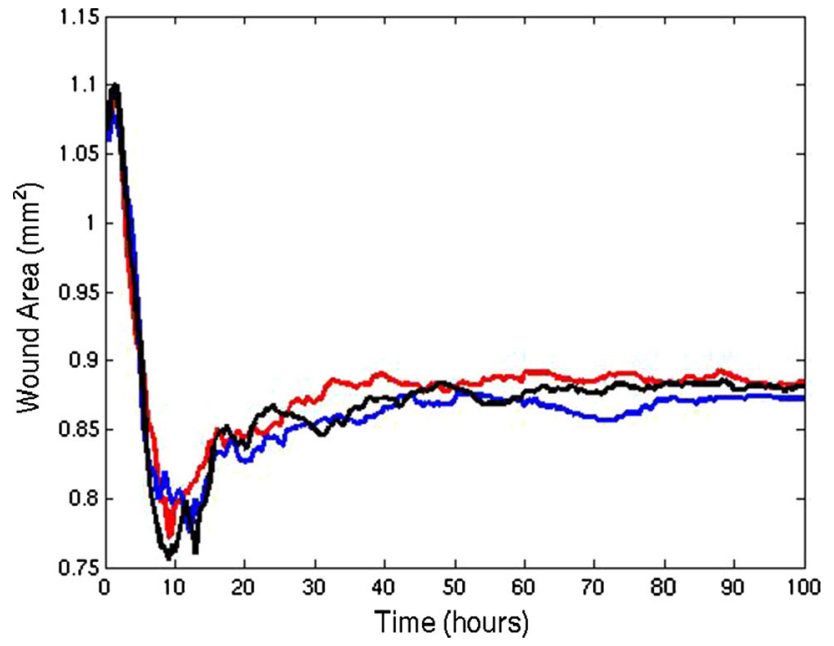

Fig. 10 The wound area as a function of time for different runs with an identical set of parameters ical cell-matrix interactions with cells determine collective endothelial cell behaviour by means of durotaxis, where cells move according to the gradient of extracellular matrix rigidity. Furthermore, cell-cell interaction in terms of communication through cellular forces significantly effects the collective cell behaviour. The model that they employ for the cell traction forces was developed by Lemmon and Romer (2010). In the formalism of (Lemmon and Romer 2010), the cellular traction is modelled by means of a grid, which coincides with the cellular Potts lattice in the study by van Oers et al. (2014), in which at each mesh point that is occupied by a cell a body-force pointing towards (or away from) the cell centre is assigned. This gives an overall displacement field over the entire substrate over which the cell is migrating, or in a three dimensional context, which is not shown in their paper, in the tissue including the cell and its surrounding extracellular matrix. Our approach differs from 
their approach in the sense, that we only consider the forces that the cell acts on its boundary onto the extracellular matrix and that our forces are taken normal to the cell boundary. This was motivated by our interest in contraction of burns where the (extracellular) region around the damage experiences traction towards the wound centre, by which we are only interested in the impact of the cells on the surroundings. Furthermore, we allow the extracellular matrix domain to deform where the boundaries are free. Our approach also gives a displacement and stress field over the domain of computation, which can be used to model intercellular contact between cells such that they can form clusters. We have similar simulations on the basis of the strain energy density using simplistic analytic relationships for the displacement over the substrate in Vermolen and Gefen (2013) and Vermolen and Gefen (2012). The present modelling provides a neat mathematical generalisation. In the present modelling, cells are allowed to migrate through a chemical stimulus, see the $\mu$-term in Eq. (22). Here, the driving force is a gradient, it could as easily include a gradient of the substrate or extracellular rigidity to model durotaxis. One could also include any mechanical signal to include cellular migration triggered by the traction of other cells such that clusters of cells can be formed. In this sense, we think that the present modelling approach could be extended and be used to model cell clustering. This is a topic of present research where we model the formation of capillary sprouts for vascularisation processes by collective migration behaviour of endothelial cells using the current cell-based approach with chemical and mechanical signalling processes as an alternative to cellular Potts modelling. Besides our cell-based approach for the processes, the present modelling approach for the computation of cell traction on the extracellular matrix through the cell boundary, is probably also useful in the context of cellular Potts modelling since if one knows the lattice points that coincide with the boundary of the cell, then one can impose a force in whatever direction one wants: either in (or against) the direction of the vector connecting the boundary point under consideration to the cell centre or in the direction orthogonal to the cell boundary. We also remark that the present approach allows an analytic treatment by the use of a superposition of Green's fundamental solutions for elasticity (this is actually how the idea came into existence). It would be interesting to investigate the relation between the integration over a cell boundary and an effective point-wise approach.

We finally realise that the present modelling studies for the contraction of burns and cyclic loadings of tissue by for instance muscle cells have a very simplified nature since the aim of the paper is to present an alternative, mathematically neat formalism for cell traction through the cell boundary on the extracellular matrix and to show the potentials of the modelling framework.

\section{Conclusions}

We propose a formalism to compute the boundary forces that are exerted by cells. The boundary forces are treated by dividing the cell boundary into boundary segments over which a delta force is assigned to each segment. Next, to these contractile forces of cells, we consider permanent forces as a set of point sources that are activated over a pseudo-time, which evolves depending on the occupancy of a control/area element by a cell of specific phenotype (in the present application, these cells are myofibroblasts). The model is applied to contraction of burns and to colorgreen cellular cyclic loading. Some parameter variation illustrates the dependence of the simulations on several biological parameters. As far as we know, the model is original in its kind.

Acknowledgments The author Fred Vermolen is grateful for the financial support by the Dutch Burns Foundation and acknowledges the discussions with the plastic surgeons Paul van Zuijlen and Frank Niessen.

Open Access This article is distributed under the terms of the Creative Commons Attribution License which permits any use, distribution, and reproduction in any medium, provided the original author(s) and the source are credited.

\section{References}

Byrne H, Drasdo D (2009) Individual-based and continuum models of growthing cell populations: a comparison. J Math Biol 58:657-687

Gefen A (2010) Effects of virus size and cell stiffness on forces, work and pressures driving membrane invagination in a receptormediated endocytosis. J Biomech Eng (ASME) 132(8):4501-4505

Graner F, Glazier J (1992) Simulation of biological cell sorting using a two-dimensional extended Potts model. Phys Rev Lett 69:20132016

Groh A, Louis AK (2010) Stochastic modeling of biased cell migration and collagen matrix modification. J Math Biol 61:617-647

Lemmon CA, Romer LH (2010) A predictive model of cell traction forces based on cell geometry. Biophys J 99:L78-L80

Merks MH, Koolwijk P (2009) Modeling morphogenesis in silico and in vitro: towards quantitative, predictive, cell-based modeling. Math Model Nat Phenom 4(4):149-171

Murray JD (2004) Mathematical biology II: spatial models and biomedical applications. Springer, New York

Neilson MP, MacKenzie JA, Webb SD, Insall RH (2011) Modeling cell movement and chemotaxis using pseudopod-based feedback. SIAM J Sci Comput 33(3):1035-1057

Olsen L, Sherratt JA, Maini PK (1995) A mechanochemical model for adult dermal wound closure and the permanence of the contracted tissue displacement role. J Theor Biol 177:113-128

Steele JM (2001) Stochastic calculus and financial applications. Springer, New York

Van Oers RFM, Rens EG, LaValley DJ, Reinhart-King CA, Merks RMH (2014) Mechanical cell-matrix feedback explains pairwise and collective endothelial cell behavior in vitro. PLoS Comput Biol 10(3):e1003774

Valero C, Javierre E, Garcia-Aznar JM, Gomez-Benito MJ (2014) A cell regulatory mechanism involving feedback between contraction and tissue formation guides wound healing progression. PLoS One 9(3):e92774. doi:10.1371/journal.pone.0092774 
Vermolen FJ, Gefen A (2012) A semi-stochastic cell-based formalism to model the dynamics of migration of cells in colonies. Biomech Model Mechanobiol 11(1-2):183-195

Vermolen FJ, Gefen A (2013) A phenomenological model for chemicomechanically induced cell shape changes during migration and cell-cell contacts. Biomech Model Mechanobiol 12:301-323
Vermolen FJ, Javierre E (2012) A finite-element model for healing of cutaneous wounds combining contraction, angiogenesis and closure. J Math Biol 65(5):967-996. doi:10.1007/ s00285-011-0487-4 